\title{
SPACE STRUCTURES PROCESSED BY THE GROUP SUPERMATRIX PROCEDURE
}

\section{A B S T R A C T}

The shapes of natural and technical space structures may have geometrical configuration with symmetry properties that can be analyzed by superior mathematical modeling of the group theory, which has been shown in this paper by some aspects and results of the Author's group supermatrix procedure using the group supermatrix chain of the symmetry groups $\mathrm{C}_{2}, \mathrm{D}_{2}, \mathrm{D}_{2 \mathrm{~h}}$ and linear, rectangular, hexahedral and icosahedral configurations. This procedure formulates the analysis of structures composed according to definite space patterns with simple and complex symmetries, as well as symmetrized nonsymmetrical patterns, providing many qualitative and quantitative advantages in comparison with conventional methods.
Đorđe Zloković

Serbian Academy of Sciences and Arts
KEY WORDS

SPACE STRUCTURES

SYMMETRY GROUPS

GROUP SUPERMATRIX PROCEDURE

GROUP CHARACTER TABLE FINITE ELEMENT ANALYSIS 


\section{INTRODUCTION}

Natural and technical space structures are in equilibrium according to the laws of mechanics, the natural shapes created by evolution of matter and the technical ones by human knowledge. Besides stability they should attain optimal use of material and performance of their functions. A hovering rotationally symmetrical soap bubble has an approximate spherical shape produced by the difference between air pressures inside and outside the bubble, and the influence of gravitation.

Repetition of basic elements according to a definite space pattern in nature, e. g. the atoms in crystals (Fig. 1), has an analogy in repeating the nodes in metal structures consisting of nodes and rods (Fig. 2).

In mathematics, physics and chemistry the term symmetry is understood as: (1) correspondence according to size, shape and arrangement of parts on opposite sides of the plane, line or point; (2) type of regularity, as in case of the circle or some other figure in the plane, which is characterized by geometrical operations, such as rotation or reflection, that leave the figure unchanged; (3) the property of the physical system which permits the system to remain unchanged under specific physical or mathematical transformations.

Structures composed of basic elements arranged according to the point patterns in space with symmetry properties can be analyzed by the group theory, a highly developed field of mathematics which treats symmetry in a systematical and all including manner. A symmetry group possesses elements as symmetry operations with the property that when applied to the structure they only permute elements of the structure without changing anything in a physical sense.
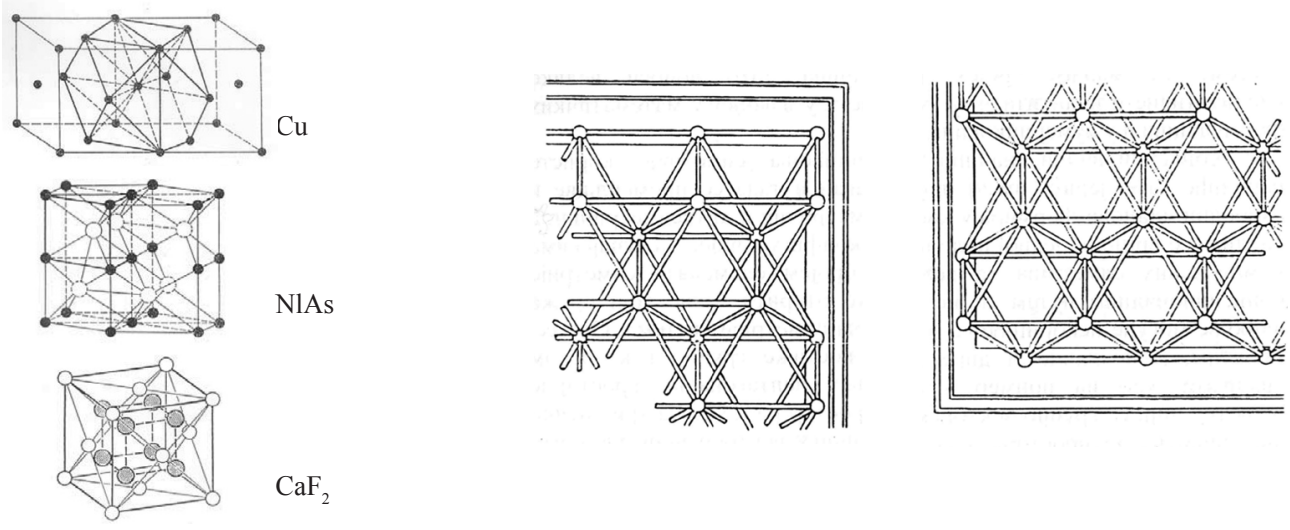
In symmetry groups symmetry elements are rotation axes, reflection planes and roto-reflections. The smallest group possesses the identity element and a reflection plane, then follow the groups with more symmetry elements and finally the icosahedral group with 120 symmetry elements.

Geometrical configurations of technical structures, composed of elements arranged in definite space patterns with simple and complex symmetries, as well as symmetrized nonsymmetrical patterns, are formulated and analyzed by the group supermatrix procedure devised and developed by Zloković $[1,2,3,4,5]$. This procedure provides, in comparison with the conventional methods, maximum utilization of symmetry properties, very compact mathematical description and drastic reductions of the amount of computation in numerical analysis.

\section{THE GROUP SUPERMATRIX CHAIN OF THE GROUPS $C_{2}, D_{2}, D_{2 h}$}

Symmetry groups $C_{2}, D_{2}, D_{2 h}$ possess the property of all their group representations being one-dimensional and in their character tables all characters having the values 1 or -1 .

The symmetry properties of the line segment are described by the symmetry group $C_{2}$ with the character table

$$
\begin{array}{l|rrr}
C_{2} & E & C_{2}^{y} & y z \\
\hline \Gamma_{1} & 1 & 1 & \mathrm{~S} \\
\Gamma_{2} & 1 & -1 & \mathrm{~A}
\end{array}
$$

while the symmetry properties of a rectangle by the symmetry group $D_{2}$ with the character table

\begin{tabular}{|c|c|c|c|c|c|c|c|c|}
\hline$D_{2}$ & $E$ & $C_{2}^{z}$ & $C_{2}^{y}$ & $C_{2}^{x}$ & $x z$ & $y z$ & & \\
\hline$\overline{\Gamma_{1}}$ & 1 & 1 & 1 & 1 & S & S & & $x^{2}, y^{2}, z^{2}$ \\
\hline$\Gamma_{2}$ & 1 & 1 & -1 & -1 & A & A & $z$ & $x y$ \\
\hline$\Gamma_{3}$ & 1 & -1 & 1 & -1 & $\mathrm{~S}$ & A & $y$ & $x z$ \\
\hline$\Gamma_{4}$ & 1 & -1 & -1 & 1 & A & $\mathrm{S}$ & $x$ & $y z$ \\
\hline
\end{tabular}

where $E$ is the identity operation, while $\mathrm{C}_{2}^{\mathrm{z}}, \mathrm{C}_{2}{ }^{\mathrm{y}}, \mathrm{C}_{2}{ }^{\mathrm{x}}$ are rotations through $180^{\circ}$ degrees about $z, y, x$ coordinate axes respectively. The irreducible representations $\Gamma_{1}, \Gamma_{2}, \Gamma_{3}, \Gamma_{4}$ of the group $D_{2}$ are designated by $\mathrm{S}$ (symmetrical) or A (antisymmetrical) to the coordinate planes $x z, y z$, as well as represented by respective Cartesian terms and products.

The symmetry properties of the rectangular hexahedron are described by the symmetry group $D_{2 h}$ with the character table 


\begin{tabular}{|c|c|c|c|c|c|c|c|c|c|c|c|c|}
\hline$D_{2 h}$ & E & $C_{2}^{z}$ & $C_{2}^{y}$ & $C_{2}^{x}$ & $i$ & $\sigma_{x y}$ & $\sigma_{x z}$ & $\sigma_{y z}$ & $x y$ & $x z$ & $y z$ & \\
\hline$\overline{\Gamma_{1}}$ & 1 & 1 & 1 & 1 & 1 & 1 & 1 & 1 & $\mathrm{~S}$ & $\mathrm{~S}$ & $\bar{S}$ & $1 x^{2}, y^{2}, z^{2}$ \\
\hline$\Gamma_{2}$ & 1 & 1 & -1 & -1 & 1 & 1 & -1 & -1 & $\mathrm{~S}$ & A & A & $x y$ \\
\hline$\Gamma_{3}$ & 1 & -1 & 1 & -1 & 1 & -1 & 1 & -1 & A & $\mathrm{S}$ & A & $x z$ \\
\hline$\Gamma_{4}$ & 1 & -1 & -1 & 1 & 1 & -1 & -1 & 1 & A & A & $\mathrm{S}$ & $y z$ \\
\hline$\Gamma_{5}$ & 1 & 1 & 1 & 1 & -1 & -1 & -1 & -1 & A & A & A & $x y z$ \\
\hline$\Gamma_{6}$ & 1 & 1 & -1 & -1 & -1 & -1 & 1 & 1 & A & $\mathrm{S}$ & $\mathrm{S}$ & $z$ \\
\hline$\Gamma_{7}$ & 1 & -1 & 1 & -1 & -1 & 1 & -1 & 1 & S & A & $\mathrm{S}$ & $y$ \\
\hline$\Gamma_{8}$ & 1 & -1 & -1 & 1 & -1 & 1 & 1 & -1 & $\mathrm{~S}$ & $\mathrm{~S}$ & A & $x$ \\
\hline
\end{tabular}

where $E$ is the identity operation, while $\mathrm{C}_{2}{ }^{\mathrm{z}}, \mathrm{C}_{2}{ }^{\mathrm{y}}, \mathrm{C}_{2}{ }^{\mathrm{x}}$ are rotations through $180^{\circ}$ degrees about $z, y, x$ coordinate axes, $i$ inversion operation, $\sigma_{x y}, \sigma_{x z}, \sigma_{y z}$ reflections in $x y, x z, y z$ coordinate planes. The irreducible representations $\Gamma_{1}$, $\Gamma_{2}, \Gamma_{3}, \Gamma_{4}, \Gamma_{5}, \Gamma_{6}, \Gamma_{7}, \Gamma_{8}$ of the group $D_{2 h}$ are designated by $\mathrm{S}$ (symmetrical) or A (antisymmetrical) to the coordinate planes $x y, x z, y z$, as well as represented by respective Cartesian terms and products.

Figure 3 provides a visual presentation of relations of character tables of symmetry groups $C_{2}, D_{2}, D_{2 h}$ that describe symmetry properties of the line segment, the rectangle and the rectangular hexahedron respectively, which may be regarded as a chain that connects the one-. two- and three-dimensional elements (1-D, 2-D, 3-D) [9,10,11].

The character tables of the groups $C_{2}, D_{2}, D_{2 h}$ are connected by addition and projection according to the common pattern from the character table of the group $C_{2}$, while unit matrices $E$ and $-E$ replace 1 and -1 in character tables with the object to transform the character tables to supermatrix form. The

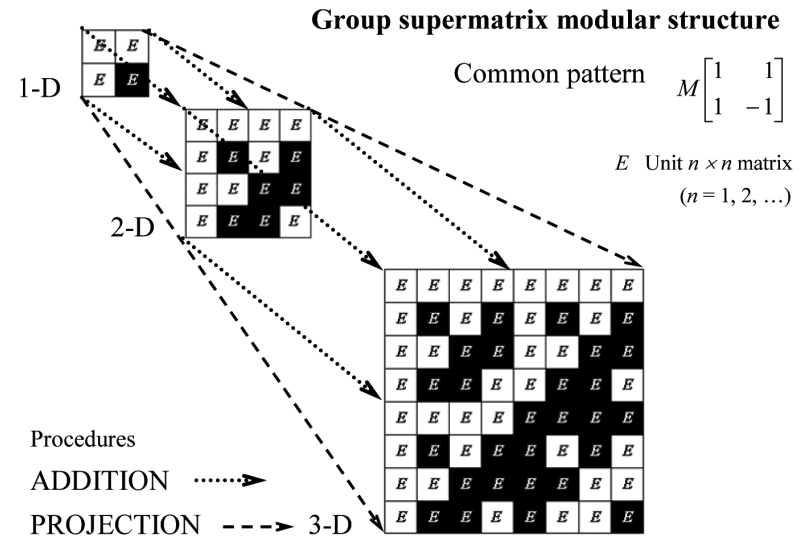

Figure 3 Relation of character tables of symmetry groups $C_{2}, D_{2}, D_{2 h}$ that describe syummetry properties of the line segment, the rectangle and the rectangular hexahedron respectively (1-D, 2-D, 3-D). Unit matrix E on white and black backgrounds designates plus and minus signs of E respectively 
unit matrix $-E$ is given on black background in order to obtain a clear visual presentation of the common pattern by

\begin{tabular}{l|l|l}
$E$ & $E$ \\
$E$ & $E$
\end{tabular}$\quad$ representing the supermatrix $\left[\begin{array}{ll}E & E \\
E & -E\end{array}\right]$.

\section{THE RECTANGULAR HEXAHEDRAL ELEMENT WITH SYMMETRY PROPERTIES DESCRIBED BY THE GROUP $D_{2 h}$}

The modular structure of the group $D_{2 h}$ makes it possible to formulate complex finite rectangular hexahedral elements using the basic eight monomial functions

$\begin{array}{llllllll}1 & x y & x z & y z & x y z & z & y & x\end{array}$

obtained from the eight symmetry types of the group representations $\Gamma_{1}, \Gamma_{2}$, $\Gamma_{3}, \Gamma_{4}, \Gamma_{5}, \Gamma_{6}, \Gamma_{7}, \Gamma_{8}$ of the group $D_{2 h}$. They are derived by the product $z^{p} y^{\prime} x^{t}$, where $p, r, t$ equal 0 or 1 when $\mathrm{S}$ (symmetry) or A (antisymmetry) designate symmetry or antisymmetry to coordinate planes $x y, x z, y z$ of the particular group representation, which is given in Table 1. The eight subspaces of the eight-node rectangular hexahedral element with three degrees of freedom of nodal displacements are shown in Fig. 4.

\begin{tabular}{|l|l|l|l|l|l|l|l|l|}
\hline Subspace & $U_{1}$ & $U_{2}$ & $U_{3}$ & $U_{4}$ & $U_{5}$ & $U_{6}$ & $U_{7}$ & $U_{8}$ \\
\hline $\begin{array}{l}\text { Symmetry to } \\
\mathrm{xy}, \mathrm{xz}, \mathrm{yz} \text { planes }\end{array}$ & SSS & SAA & ASA & AAS & AAA & ASS & SAS & SSA \\
\hline $\begin{array}{l}\text { Replacing } \\
\mathrm{S}, \text { A by } 0,1\end{array}$ & 000 & 011 & 101 & 110 & 111 & 100 & 010 & 001 \\
\hline $\mathrm{z}^{\mathrm{p}} \mathrm{y}^{\mathrm{r}} \mathrm{x}^{\mathrm{t}}$ & $\mathrm{z}^{0} \mathrm{y}^{0} \mathrm{x}^{0}$ & $\mathrm{z}^{0} \mathrm{y}^{1} \mathrm{x}^{1}$ & $\mathrm{z}^{1} \mathrm{y}^{0} \mathrm{x}^{1}$ & $\mathrm{z}^{1} \mathrm{y}^{1} \mathrm{x}^{0}$ & $\mathrm{z}^{1} \mathrm{y}^{1} \mathrm{x}^{1}$ & $\mathrm{z}^{1} \mathrm{y}^{0} \mathrm{x}^{0}$ & $\mathrm{z}^{0} \mathrm{y}^{1} \mathrm{x}^{0}$ & $\mathrm{z}^{0} \mathrm{y}^{0} \mathrm{x}^{1}$ \\
\hline Functions & 1 & $x y$ & $x z$ & $y z$ & $x y z$ & $Z$ & $y$ & $X$ \\
\hline
\end{tabular}

Table 1 Derivation of functions pertaining to particular representations of the group $\mathrm{D}_{2 \mathrm{~h}}$ in the subspaces $\mathrm{U}_{1}, \mathrm{U}_{2}, \mathrm{U}_{3}, \mathrm{U}_{4}, \mathrm{U}_{5}, \mathrm{U}_{6}, \mathrm{U}_{7}, \mathrm{U}_{8}$

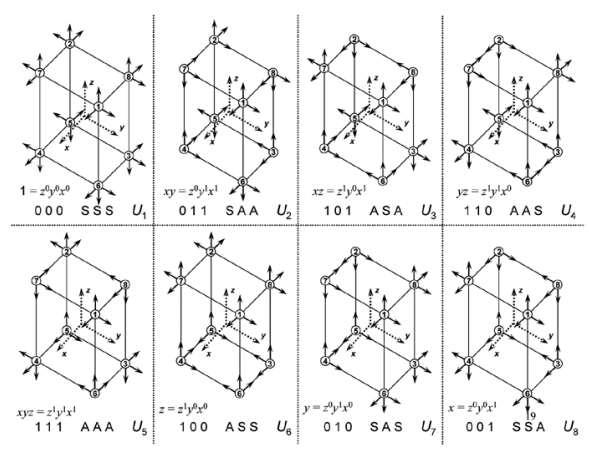

Figure 4 The eight-node rectangular hexahedral element with three degrees of freedom in the nodes in eight G-invariant subspaces of the group $\mathrm{D}_{2 \mathrm{~h}}$

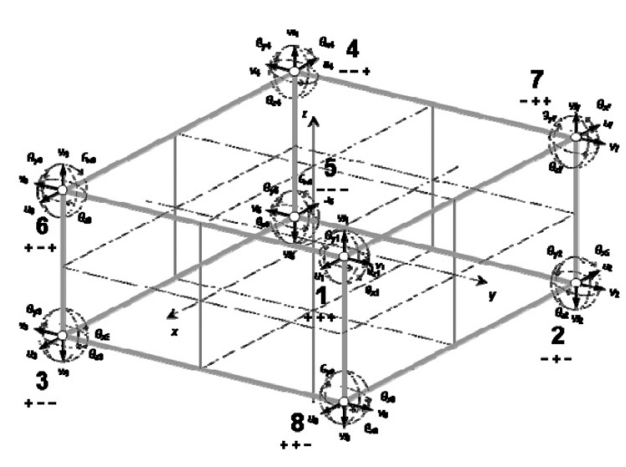

Figure 5 Group supermatrix structuring of the rectangular hexahedral element with $48 \mathrm{~d}$. o. f. 
The nodal numbering of the eight-node rectangular hexahedral element is accomplished by designating the node in the first octant by 1 and then applying particular symmetry operations according to their sequence at the top of the character table. Thus, application of the identity operation $E$ (rotation through $0^{\circ}$ ) leaves number 1 in its place and the following numbering by $2,3,4,5,6$, 7,8 is accomplished by applying on node 1 rotations $C_{2}^{z}, C_{2}^{y}, C_{2}^{x}$, inversion $i$ and reflections $\sigma_{x y}, \sigma_{x z}, \sigma_{y z}$.

In the local coordinate systems the directions of displacements of the nodes suit the symmetry type of each subspace given by the group representations in the character table. This is different in comparison with the conventional procedure, where positive directions of the axes of local coordinate systems in the nodes all coincide with positive directions of coordinate axes of the global coordinate system $[12,13,15]$.

In Fig. 5 the first subspace of the eight-node rectangular hexahedral element with six degrees of freedom in each node (three displacements and three rotations) is shown. This subspace is symmetrical with respect to all coordinate planes $x y, x z, y z$ and the directions of displacements and rotations in the nodes are in accordance with the symmetry type of the first subspace.

All influences on the element (forces, moments, displacements, rotation, temperature on all nodes) can be performed from the first octant of the global coordinate system using the boundary conditions in the coordinate planes $x y$, $x z, y z$ for particular subspaces (Fig. 6).

The shape functions of the 64-node rectangular hexahedral element in Fig. 7 can be derived using the eight monomial functions $1, x y, x z, y z, x y z, z, y, x$ shown earlier.

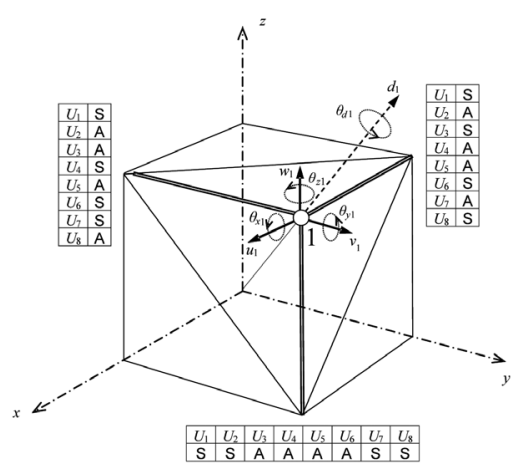

Figure 6 First octant of the eight-node rectangular hexahedral element with boundary conditions in the coordinate planes $x y, x z, y z$ (S - symmetry A - antisymmetry)

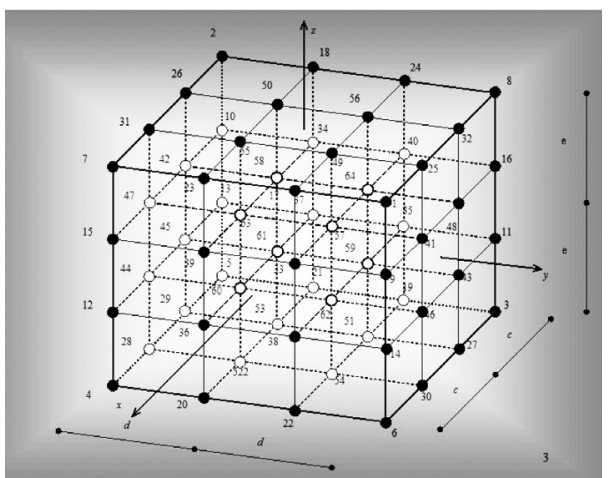

Figure 7 64-node rectangular hexahedral finite element with the group supermatrix numbering eight nodal sets, each set containing eight nodes 


\section{SPHERICAL NETWORK WITH SYMMETRIES DESCRIBED BY THE ICOSAHEDRAL GROUP $I_{h}$}

The spherical network is circumscribed around the regular dodecahedron and the regular icosahedron (Fig. 8). Twofold rotation axes emerge from midpoints of edges of the icosahedron, while threefold axes are perpendicular to the triangular faces of the icosahedron, with fivefold axes passing through its vertices [14]. The nodal types in the icosahedral spherical network are explained in Table 2.

\begin{tabular}{|c|c|l|}
\hline Nodal type & Designation & Position of the node \\
\hline 1 & - & node in a vertex of the inscribed icosahedron \\
\hline 2 & $\bigcirc$ & node in a vertex of the inscribed dodecahedron \\
\hline 3 & $\times$ & node in a midpoint of the spherical triangle $T_{20}$ \\
\hline 4 & $\bullet$ & node at the contour of the spherical triangle $T_{120}$ \\
\hline 5 & 0 & node in the interior of the spherical triangle $T_{120}$ \\
\hline
\end{tabular}

Table 1 Derivation of functions pertaining to particular representations of the group D2h in the subspaces $\mathrm{U} 1, \mathrm{U} 2, \mathrm{U} 3, \mathrm{U} 4, \mathrm{U} 5, \mathrm{U} 6, \mathrm{U} 7, \mathrm{U} 8$

The icosahedral group $I_{h}$ contains 10 classes of conjugate elements

$$
\begin{array}{lllll}
E & 12 C_{5} & 12 \mathrm{C}_{5}{ }^{2} & 20 C_{3} & 15 C_{2} \\
i & 12 S_{10}{ }^{2} & 12 S_{10} & 20 S_{6} & 15 \sigma
\end{array}
$$

$E \quad-$ the identity operation

$12 C_{5} \quad$ - rotations $C_{5}, C_{5}^{-1}$ by $72^{\circ},-72^{\circ}$ (2 rotations about 6 axes through the vertices of the icosahedron) $12 C_{5}^{2}-$ rotations $C_{5}^{2}, C_{5}^{-2}$ by $144^{\circ},-144^{\circ}$ (2 rotations about 6 axes through the vertices of the icosahedron)

$20 C_{3} \quad$ rotations $C_{3}, C_{3}{ }^{1}$ by $120^{\circ},-120^{\circ}$ (2 rotations about 10 axes through the faces of the icosahedron)

$15 C_{2}$ - rotations $C_{2}$ by $180^{\circ}$ (one rotation about 15 axes through midpoints of the edges of the icosahedron)

$i \quad-$ the inversion operation

$12 S_{10}{ }^{2}$ - roto-reflections $S_{10}{ }^{2}, S_{10}^{-2}$ by $72^{\circ},-72^{\circ}$ (2 roto-reflections about 6 axes)

$12 S_{10} \quad$ - roto-reflections $S_{10}, S_{10}{ }^{-1}$ by $36^{\circ},-36^{\circ}$ ( 2 roto-reflections about 6 axes)

$20 S_{6}$ - roto-reflections $S_{6}, S_{6}^{-1}$ by $60^{\circ},-60^{\circ}$ (2 roto-reflections about 10 axes)

$15 \sigma-15$ reflections in 15 symmetry planes

In total: 120 symmetry operations 
The character table of the group $I_{h}$ is

\begin{tabular}{r|rrrrrrrrrr}
$I_{h}$ & $E$ & $12 C_{5}$ & $12 C_{5}^{2}$ & $20 C_{3}$ & $15 C_{2}$ & $i$ & $12 S_{10}^{2}$ & $12 S_{10}$ & $20 S_{6}$ & $15 \sigma$ \\
\hline$A_{g}$ & 1 & 1 & 1 & 1 & 1 & 1 & 1 & 1 & 1 & 1 \\
$T_{1 g}$ & 3 & $\varphi$ & $-\frac{1}{\varphi}$ & 0 & -1 & 3 & $\varphi$ & $-\frac{1}{\varphi}$ & 0 & -1 \\
$T_{2 g}$ & 3 & $-\frac{1}{\varphi}$ & $\varphi$ & 0 & -1 & 3 & $-\frac{1}{\varphi}$ & $\varphi$ & 0 & -1 \\
$G_{g}$ & 4 & -1 & -1 & 1 & 0 & 4 & -1 & -1 & 1 & 0 \\
$H_{g}$ & 5 & 0 & 0 & -1 & 1 & 5 & 0 & 0 & -1 & 1 \\
$A_{1 u}$ & 1 & 1 & 1 & 1 & 1 & -1 & -1 & -1 & -1 & -1 \\
$T_{1 u}$ & 3 & $\varphi$ & $-\frac{1}{\varphi}$ & 0 & -1 & -3 & $-\varphi$ & $\frac{1}{\varphi}$ & 0 & 1 \\
$T_{2 u}$ & 3 & $-\frac{1}{\varphi}$ & $\varphi$ & 0 & -1 & -3 & $\frac{1}{\varphi}$ & $-\varphi$ & 0 & 1 \\
$G_{u}$ & 4 & -1 & -1 & 1 & 0 & -4 & 1 & 1 & -1 & 0 \\
$H_{u}$ & 5 & 0 & 0 & -1 & 1 & -5 & 0 & 0 & 1 & -1
\end{tabular}

where $\phi=1 / 2(\sqrt{ } 5+1)$ is the known value of the golden section.

Application of the icosahedral group $I_{h}$ with 120 symmetry operations decomposes the space of the spherical network into 10 G-invariant subspaces with their specific symmetry types.

Spherical networks are generated by applying symmetry operations of the group on the elementary part of the network.

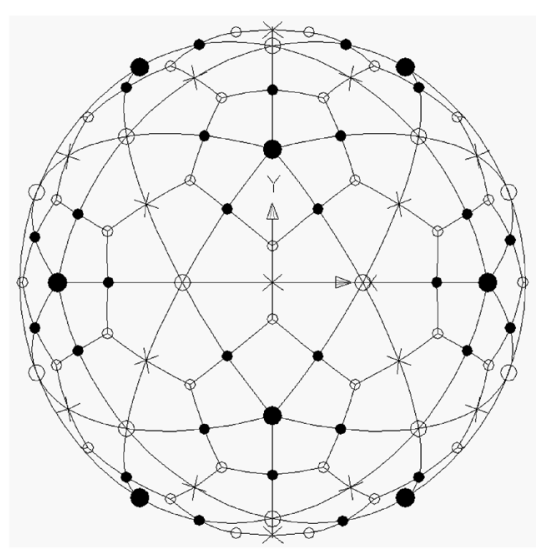




\section{CONCLUSION}

The group supermatrix procedure is formulated for a large variety of applications in the analysis of structures in architectural, civil and mechanical engineering $[5,6,7,8]$, with many computed examples that show a distinct superiority in regard to conventional utilization of symmetry.

In the Author's books and papers the group supermatrix procedure is developed in:

- structural analysis: vibration, stability and statics;

- finite element analysis;

- symmetrical and nonsymmetrical structures;

- complex spherical networks;

- vibration of cable networks;

- modular structuring,

and other modeling of structural shapes.

1 Zloković, G., "Group theory and G-vector spaces in structural analysis: vibration, stability and statics", Ellis Horwood / John Wiley, Chichester, United Kingdom, 1989. Schuster, Chichester, United Kingdom, 1992. engineering structures", in "Structural Engineering Review", Elsevier, Edinburgh, United Kingdom 6(1), 39-50, 1994. and hexahedral isoparametric finite elements in mechanics", in "Solid Mechanics", Serbian Academy of Sciences and Arts, Beograd, Serbia, 223-232, 1997. systems by the group supermatrix procedure”, in “Computers \& Structures”, Edinburgh, United Kingdom, 71, 637-649, 1999. mechanics", National Technical University, Athens, Greece, 2000. 
Zloković, G., "Vibration of cable networks formulated in G-invariant subspaces", in "Bulletin T.CXXVI de l'Académie serbe des sciences et des arts", Classe des Sciences techniques N ${ }^{\circ} 29$, 81-97, Beograd, Serbia, 2003.

Zloković, G., Maneski, T., Nestorović, M., "Group theoretical formulation of quadrilateral and hexahedral isoparametric finite elements", in "Computers \& Structures", Edinburgh, United Kingdom, 82, 883-899, 2004.

Zloković, G., "Group supermatrix modular structure developed on properties and relations of symmetry groups C2, D2, D2h", in "Bulletin T.CXXXII de l'Académie serbe des sciences et des arts", Classe des Sciences techniques No 30, 61-89, Beograd, Serbia, 2006.

Zloković, G., "Group supermatrix modular structuring by symmetry groups D2 and D2h", in "Bulletin T.CXXXII de l'Académie serbe des sciences et des arts", Classe des Sciences techniques No 30, 91-102, Beograd, Serbia, 2006.

Zloković, G., "The modular group supermatrix procedure in structural analysis", The Eleventh International Conference on Civil, Structural and Environmental Engineering Computing, CD Paper $\mathrm{N}^{\circ} 135$, pp. 1-20, St. Julians, Malta, 2007.

Zloković, G., "The shape functions of finite elements in the modular group supermatrix procedure, in "Bulletin T.CXXXIII de l'Académie serbe des sciences et des arts", Classe des Sciences techniques No 31, 1-45, Beograd, Serbia, 2009.

Zloković, G., "The novel derivation of shape functions of the 64-node rectangular hexahedral finite element by the group supermatrix procedure, in "Bulletin T.CXXXIII de l'Académie serbe des sciences et des arts", Classe des Sciences techniques № 31, 47-65, Beograd, Serbia, 2009.

Zloković, G., "Analysis and Generation of Complex Spherical Networks Based on Octahedral and Icosahedral Symmetry Groups", moNGeometrija 2010, 25th National and 2nd International Scientific Conference, CD pp. 1-18, Faculty of Mechanical Engineering, University of Belgrade. 2010.

Zloković, G., "The novel derivation of shape functions of finite elements by the group supermatrix procedure, in "Bulletin T.CXLII de l'Académie serbe des sciences et des arts", Classe des Sciences techniques No 32, 1-46, Beograd, Serbia, 2010. 